\title{
New ASCA Observations of two Anomalous X-ray Pulsars
}

\author{
B. Paul ${ }^{1}$, M. Kawasaki, T. Dotani, and F. Nagase \\ Institute of Space and Astronautical Science \\ 3-1-1 Yoshinodai, Sagamihara, Kanagawa 229-8510, Japan
}

\begin{abstract}
New ASCA observations of two anomalous X-ray pulsars (AXP) 4U 0142+61 and 1E 1048.1-5937, made in 1998, when compared to earlier observations in 1994 show remarkable stability in the intensity, spectral shape and pulse profile. The energy spectra consist of two components, a power-law and a blackbody emission from the neutron star surface. In 1E 1048.1-5937, we have identified three epochs with different spin-down rates and discuss its implications for the magnetar hypothesis of the AXPs. We also note that the spin-down rate and its variations in IE 1048.1-5937 are much larger than what normally can be produced by an accretion disc with very low mass accretion rate corresponding to its low X-ray luminosity.
\end{abstract}

\section{Introduction}

A set of X-ray pulsars are known to have remarkable similarity in their properties which are different from other binary or isolated X-ray pulsars (Mereghetti \& Stella 1995). The properties common to most of these objects are a) pulse period in a small range of $5-12 \mathrm{~s}$, b) monotonous spin down with $\dot{\mathrm{P}}$ in the range of, $5 \times 10^{11}-1.3 \times 10^{13} \mathrm{~s}, \mathrm{c}$ ) identical $\mathrm{X}$-ray spectrum consisting of steep power-law $(\Gamma=3-4)$ and black body component $(\mathrm{kT} \sim 0.5 \mathrm{keV})$, d) stable X-ray luminosity $\left(10^{34}-10^{36}\right.$ ergs $\left.^{-1}\right)$ for years, e) faint or unidentified optical counterpart, and $f$ ) no evidence of orbital motion. The sources also have a galactic distribution, most of these are within $|b| \leq 0.5^{\circ}$ and all are probably young $\left(\sim 10^{4} \mathrm{yr}\right)$ because of their association with SNR or molecular clouds. The objects in which all the properties mentioned above have been observed are $4 \mathrm{U} 0142+61,1 \mathrm{E} 2259+586,1 \mathrm{E}$ 1048.1-5937, 1RXS J170849.0-400910 and 1E 1841-045 (Les 73).

Considering the strong similarity between these handful of sources, it has been proposed that they have same physical nature and different scenarios have been proposed to explain the observed properties. The prominent models are accretion from low mass binary companion (Mereghetti \& Stella 1995), single neutron star accreting from molecular cloud or remnant of common envelope evolution (van Paradijs, Taam \& van den Heuvel 1995; Ghosh, Angelini \& White

\footnotetext{
${ }^{1}$ On leave from the Tata Institute of Fundamental Research, Homi Bhabha Road, Colaba, Mumbai 400005, India
} 
1997) and extremely high magnetic filed neutron star radiating due to magnetic field decay (Thompson \& Duncan 1996). Unlike the radio pulsars and rotationally powered X-ray pulsars, in the AXPs, the spin-down rate is not large enough to power the observed X-ray emission. The AXPs are in many respect also similar to the X-ray counterparts of the Soft Gamma-ray Repeaters (SGR).

Stability of the $\mathrm{X}$-ray emission properties (spin-down rate, luminosity, spectral shape and pulse shape and fraction) is usually mentioned as one important aspect of the AXPs though one has to compare between observations made with different instruments for which the energy band, energy resolution and sensitivity are not identical. To make a rigorous comparison in the stability of the X-ray emission properties we have studied two AXPs with the ASCA with four years time difference between the observations. The aim was to critically examine the stability of the X-ray emission pattern and more pulse period measurements which may provide support to either the accretion powered or the magnetar hypothesis for these objects.

\section{Observations and results}

Both the sources were observed twice with $A S C A$, in $1994 \& 1998$ with about 4 years time difference between the two observations. The 1998 observations resulted in new measurement of pulse periods $8.688267 \pm 0.000024$ (4U 0142+61, epoch $\mathrm{MJD}=51046.7)$ and $6.450815 \pm 0.000002(1 \mathrm{E} 1048.1+5937$, epoch MJD $=51021.1$ ). The pulse fraction, defined as ratio of the pulsed to total flux was calculated from background subtracted pulse profiles in the $0.5-10.0 \mathrm{keV}$ band. Pulse fractions ( $\sim 9 \%$ and $\sim 75 \%$ in $4 \mathrm{U} 0142+61$ and $1 \mathrm{E} 1048.1-5937$ respectively) and pulse shapes were found to be identical in both the observations. Light curves of the two sources did not show any intensity variations at minutes to days time scale. The pulse period measurements of $4 \mathrm{U} 0142+61$ is rather scarce except for the last two years (Figure 1) and is consistent with a constant spin-down rate. The flux history of $4 \mathrm{U} 0142+61$ shows only about $15 \%$ variability around the average value, and multiple measurements with the same instrument of $A S C A$ and Beppo-SAX (Israel et al. 1999) gave almost identical flux. In the source 1E 1048.1-5937, including the 1998 ASCA observation (Figure 1), three different epochs with different spin-down rates of $1.5 \pm 0,5 \times 10^{-11}$, $1.67 \times 10^{-11}$, and $3.29 \times 10^{-11} \mathrm{~s} \mathrm{~s}^{-1}$ are clearly identified. In $1 \mathrm{E} 1048.1-5937$, the over all intensity during the two $A S C A$ observations and one Beppo-SAX observation in between are within $10 \%$ of the average value. The flux measurements from the previous observations are about a factor 3 higher than the recent measurements. But for non-imaging instruments, contribution from the nearby bright source $\eta$-Carina may have resulted in overestimation of the flux.

For both the sources, a spectral model consisting a power-law and a blackbody component with line of sight absorption results in acceptable fit. For $4 \mathrm{U} 0142+61$, the power-law photon index is 3.8 and temperature of the black body component is $0.39 \mathrm{keV}$. Same for $1 \mathrm{E} 1048.1-5937$ are 3.0 and $0.56 \mathrm{keV}$ respectively. For details of the spectral fitting and values of all the spectral parameters for the two observations, see Paul et al. (1999). In the ASCA observations of both the sources separated by 4 years we have found that the overall intensity and spectral parameters have remarkable stability. 

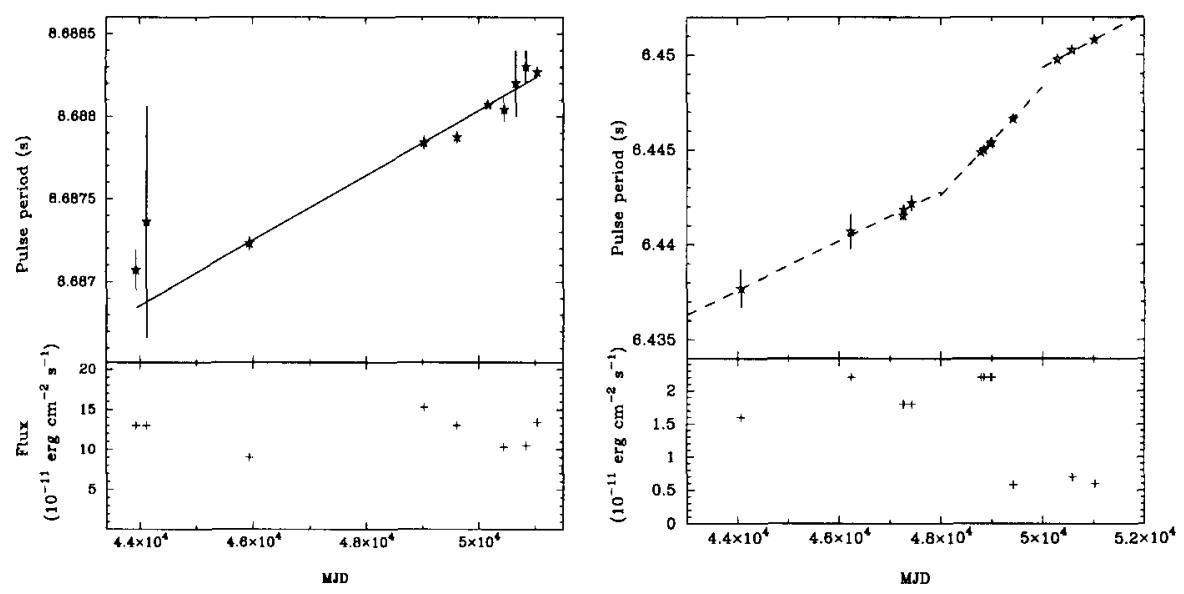

Figure 1. Pulse period and flux history of $4 \mathrm{U} 0142+61$ and $1 \mathrm{E}$ 1048.1-5937. The pulse period measurements with $A S C A$ are marked with filled symbols.

\section{Accretion torque in the common envelope evolution model}

In the common-envelope evolution model of the AXPs, the pulsar is rotating near its equilibrium period. The spin-down is explained with the assumption that the mass accretion rate from the disc decreases slowly on viscous time scale with corresponding increase in the equilibrium period. For $1 \mathrm{E} 1048.1-5937$, which has a pulse period of $6.5 \mathrm{~s}$ and luminosity of $6.3 \times 10^{33} \mathrm{erg} \mathrm{s}^{-1}$ for a distance of $3 \mathrm{kpc}$ (or in a more favourable case, $7 \times 10^{34} \mathrm{erg} \mathrm{s}^{-1}$, if the source is at a distance of $10 \mathrm{kpc}$ ), even if we assume that all of the X-ray emission is a result of disc accretion, the accretion torque is only $1.1 \times 10^{31}$ (or $1.2 \times 10^{32}$ ) $\mathrm{gm} \mathrm{cm}^{2}$ $\mathrm{s}^{-2}$. To achieve the observed spin-down rate, the negative torque required to be imparted onto the neutron star is $I \dot{\Omega}=4.9 \times 10^{33} \mathrm{gm} \mathrm{cm}^{2} \mathrm{~s}^{-2}$. This is a factor of 450 (or 40) larger than the accretion torque, and in the common envelope evolution model, a negative dimensionless torque of this magnitude is required to spin-down the pulsar at the observed rate. In other words, the spin-down rate of this source is much larger than what can be achieved with disc accretion onto a neutron star with a luminosity of less than $10^{35} \mathrm{erg} \mathrm{s}^{-1}$.

\section{Magnetar model}

$1 \mathrm{E} 1048.1-5937$ shows clear deviation from a constant spin-down. Two scenarios have been proposed which can explain the changing spin-down rate even when the overall braking is due to the ultra-strong magnetic filed. Melatos (1999) showed that for reasonable neutron star parameters, a radiative precession effect may take place which can give the observed spin-down variations with time scale of about 10 years. Heyl and Hernquist (1999) have proposed that the spin-down variations can be explained as glitches (similar to radio pulsars) superposed on constant spin-down. A clustering of the pulse period of 10 sources ( 7 AXPs and 

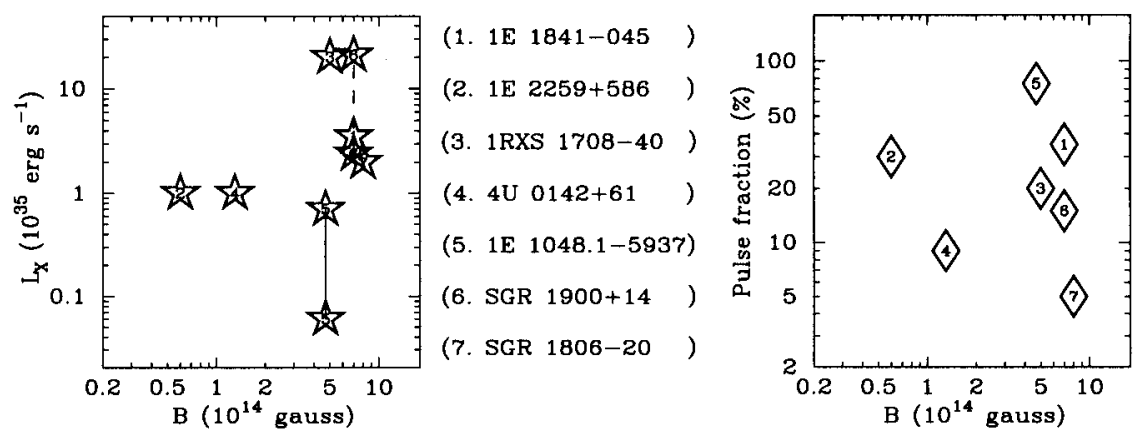

Figure 2. The X-ray luminosity and pulse fraction of the AXPs and SGRs are plotted against the magnetic field strength assuming that these objects are magnetars. Intensity variations and uncertainty in distance are shown by multiple points for some objects.

3 SGRs) in the 5-12 s range also needs to be addressed, when the magnetars are expected to be alive in X-ray until they have slowed down to a pulse period of about 70 s (Duncan \& Thompson 1992).

If the pulsation is due to confinement of the heat in the magnetic polar regions by the magnetic field, a correlation between magnetic field strength and pulse fraction is possible. This will be somewhat smeared by the the orientation of the spin and magnetic axes with respect to the line of sight of individual sources. In the magnetar model, there are two mechanisms by which X-rays can be generated. If the $\mathrm{X}$-ray emission is powered by decaying magnetic filed, $\mathrm{L}_{\mathrm{x}} \propto \mathrm{B}^{4}$ (Thompson \& Duncan 1996). Alternately, if X-ray generation is due to particle acceleration by Alfven waves resulting from small scale fracture of the crust, $\mathrm{L}_{\mathrm{x}} \propto \mathrm{B}^{2}$. The $\mathrm{X}$-ray luminosity and pulse fraction of five confirmed AXPs and two SGR sources are shown in Figure 2. against the magnetic filed strength. Though there is uncertainty in the luminosity of some sources, a 2nd or 4 th power correlation between $L_{X}$ and $B$ does not seem to be present. There is also no correlation between pulse fraction and the magnetic field.

\section{References}

Duncan, R. C., \& Thompson, C. 1992, ApJ, 392, L9

Ghosh, P., Angelini, L., \& White, N. E. 1997, ApJ, 478, 713

Heyl, J. S., \& Hernquist, L. 1999, MNRAS, 304, L37

Melatos, A. 1999, ApJ, 519, L77

Mereghetti, S., \& Stella, S. 1995, ApJ, 442, L17

Israel, G. L., et al., 1999, A\&A, 346, 929

Paul, B., Kawasaki, M., Dotani, T., \& Nagase, F. 1999, ApJ(in preparation)

Thompson, C. \& Duncan, R. C. 1996, ApJ, 473, 322

van Paradijs, J., Taam, R. E., \& van den Heuvel, E. P. J. 1995, A\&A, 299, L41 